USTC-ICTS-10-01

\title{
On M-Theory Embedding of Topologically Massive Gravity
}

\author{
H. Lü ${ }^{\dagger \ddagger}$ and Zhao-Long Wang * \\ ${ }^{\dagger}$ China Economics and Management Academy \\ Central University of Finance and Economics, Beijing 100081 \\ ${ }^{\ddagger}$ Institute for Advanced Study, Shenzhen University, Nanhai Ave 3688, Shenzhen 518060 \\ *Interdisciplinary Center for Theoretical Study, \\ University of Science and Technology of China, Hefei 230026
}

\begin{abstract}
$\underline{\text { ABSTRACT }}$
We show that topologically massive gravity can be obtained by the consistent KaluzaKlein reduction from recently constructed seven-dimensional gravity with topological terms. The internal four-manifold should be Einstein with the Pontryagin four-form constantly proportional to the volume form. We also discuss the possible lift of the system to $D=11$. This enables us to connect the mass parameter $\tilde{\mu}$ in $D=3$ to the M5-brane charge. The dimensionless quantity $3 /(G \tilde{\mu})$ is discrete and proportional to $N$, where $N$ is the number of M5-branes.
\end{abstract}


Although string theory remains to be the most promising candidate for quantizing gravity, its whole package has been proved to be difficult to study. Three-dimensional pure gravity has thus been constantly attracting people's attention as an intriguing, tantalizing and much simpler model. The Einstein-Hilbert action provides no propagating degree of freedom; however, when a negative cosmological constant is introduced, the theory admits a non-trivial BTZ black hole [1]. In the 80's topologically massive gravity (TMG), a higherderivative theory, was constructed and it contains one massive particle at the price that the sign of the Einstein-Hilbert action has to be reversed [2]. The sign reversal however would imply that the mass of the BTZ black hole turns negative. It was shown that the massive graviton disappears for certain choice of the cosmological constant and the mass parameter [3]. More three-dimensional gravities with higher but finite derivatives were recently constructed and shown to be unitary [4, [5].

There may indeed exist self-contained three-dimensional quantum gravities owing to these unusual properties. It is nevertheless of great interest to embed them in string theory. It was demonstrated that the quantity $c_{L}-c_{R}=3 /(G \tilde{\mu})$ for TMG does not change under the holographic RG flow [6]. This result was further confirmed by the $\beta$-function calculation [7]. This strongly suggests that there is a quantization condition for the dimensionless coupling $G \tilde{\mu}$. However, it was known that the homotopy condition associated with the Lorentz ChernSimons term in three dimensions is trivial and it gives rise to no quantization condition for $G \tilde{\mu}[2]$.

We expect that such a quantization condition has an origin in higher dimensions. Indeed, in a seven-dimensional gravity theory with topological terms, recently constructed in [8], the analogous coupling constant is quantized owing to the non-trivial homotopy condition in $D=7$.

In this paper, we show that TMG can in fact be obtained by the consistent Kaluza-Klein reduction from seven-dimensional gravity with topological terms. The quantity $G \tilde{\mu}$ can then be related to the corresponding one in $D=7$, and hence becomes quantized. Topological terms in $D=7$ arise naturally in supergravity coming from the $S^{4}$ reduction from M-theory, associated with the anomaly cancelation terms. This may provide a connection of the mass parameter in $D=3$ to the M5-brane charge, which is quantized. We shall first demonstrate the embedding of $D=3$ in $D=7$, and then discuss the embedding in $D=11$. We shall finish the paper with a conclusion and further discussions.

The action for topological gravity in seven dimensions is given by [8].

$$
S=\frac{1}{2 \kappa_{7}^{2}} \int\left((R-2 \Lambda) * \mathbb{1}+\mu \Omega_{1}^{(7)}+\nu \Omega_{2}^{(7)}\right),
$$


where

$$
\begin{aligned}
& \Omega_{1}^{(7)}=\Omega^{(3)} \wedge \operatorname{Tr}\left(\Theta^{2}\right), \quad \Omega^{(3)}=\operatorname{Tr}\left(d \Gamma \wedge \Gamma-\frac{2}{3} \Gamma^{3}\right) \\
& \Omega_{2}^{(7)}=\operatorname{Tr}\left(\Theta^{3} \wedge \Gamma+\frac{2}{5} \Theta^{2} \wedge \Gamma^{3}+\frac{1}{5} \Theta \wedge \Gamma^{2} \wedge \Theta \wedge \Gamma+\frac{1}{5} \Theta \wedge \Gamma^{5}+\frac{1}{35} \Gamma^{7}\right) .
\end{aligned}
$$

Here, $\Theta$ is the curvature 2-form, defined as $\Theta \equiv d \Gamma-\Gamma \wedge \Gamma$. In this paper, for a form $X$, the notation $X^{n}$ denotes the wedge product of $n X^{\prime}$ 's. The $\Omega_{i}^{(7)}$ 's are related to the Pontryagin eight-form by

$$
P^{(8)}=\frac{1}{128 \pi^{4}}\left(d \Omega_{1}^{(7)}-2 d \Omega_{2}^{(7)}\right) .
$$

The equations of motion are given by [8]

$$
R^{i j}-\frac{1}{2} g^{i j} R+\Lambda g^{i j}+C_{1}^{i j}+C_{2}^{i j}=0,
$$

where

$$
\begin{aligned}
C_{1}^{i j} & =\frac{\delta S_{1}}{\sqrt{g} \delta g_{i j}}=\frac{\mu}{4 \sqrt{g}}\left[\epsilon^{i j_{1} j_{2} j_{3} j_{4} j_{5} j_{6}}\left(R_{i_{2} j_{1} j_{2}}^{i_{1}} R_{i_{1} j_{3} j_{4}}^{i_{2}} R_{j_{5} j_{6}}^{j k}\right)_{; k}+i \leftrightarrow j\right], \\
C_{2}^{i j} & =\frac{\delta S_{2}}{\sqrt{g} \delta g_{i j}}=\frac{\nu}{4 \sqrt{g}}\left[\epsilon^{i j_{1} j_{2} j_{3} j_{4} j_{5} j_{6}}\left(R_{i_{1} j_{1} j_{2}}^{k} R_{i_{2} j_{3} j_{4}}^{i_{1}} R_{j_{5} j_{6}}\right)_{; k}+i \leftrightarrow j\right] .
\end{aligned}
$$

We now perform the Kaluza-Klein dimensional reduction on an internal four-space with fixed breathing mode. We first consider the case with positive cosmological constant and let $2 \Lambda=30 \lambda^{2}$. The metric ansatz is

$$
d s_{7}^{2}=d s_{3}^{2}+\frac{1}{\lambda^{2}} d s_{4}^{2} .
$$

We find that the consistency of the equations of motion requires that $d s_{4}^{2}$ is an Einstein space with $R_{i j}=6 g_{i j}$. Furthermore, the Pontryagin four-form in $d s_{4}^{2}$ satisfies

$$
P^{(4)} \equiv \frac{1}{8 \pi^{2}} \operatorname{Tr}(\Theta \wedge \Theta)=c \mathcal{V}_{(4)},
$$

where $\mathcal{V}_{(4)}$ is the volume form for $d s_{4}^{2}$ and $c$ has to be a constant. It is worth emphasizing that these conditions on the internal four-space can be easily missed if one merely substitutes the ansatz into the action. The condition (7) is particularly strong, but it can be satisfied. For example, homogeneous spaces clearly satisfy (7).

Let $\eta$ denote the Pontryagin instanton number, defined by

$$
\eta=\int P^{(4)}
$$

We find that the effective action in three dimensions is given by

$$
S=\frac{1}{2 \kappa_{3}^{2}} \int\left(\left(R-6 \lambda^{2}\right) * \mathbb{1}+\frac{1}{2 \tilde{\mu}} \Omega^{(3)}\right),
$$


where

$$
\frac{1}{\tilde{\mu}}=\frac{32 \pi^{2} \lambda^{4} \eta \mu}{V_{4}}
$$

Here $V_{4}$ is the volume of $d s_{4}^{2}$ and $\kappa_{3}^{2}=\lambda^{4} \kappa_{7}^{2} / V_{4}$. Note that the three-dimensional Newton's constant is given by $2 \kappa_{3}^{2}=16 \pi G$. We would like to emphasize again that only one-half of the coupling $1 / \tilde{\mu}$ can be obtained if one merely substitutes the ansatz into the sevendimensional action. Now we arrive at the action of topologically massive gravity in $D=3$. The $(\mathrm{A}) \mathrm{dS}_{3}$ length is given by

$$
\frac{1}{\ell}=\sqrt{3} \lambda
$$

The coupling constant of the topological term depends on the seven-dimensional coupling constant and the topology of the internal spaces. For the round $S^{4}$, we have $\eta=0$ and hence the topological term vanishes. As another example, let us consider the "round" $\mathbb{C P}^{2}$ with the desired cosmological constant. The metric is given by

$$
d s^{2}=d \xi^{2}+\frac{1}{4} \sin ^{2} \xi \cos ^{2} \xi(d \psi+\cos \theta d \phi)^{2}+\frac{1}{4} \sin ^{2} \xi\left(d \theta^{2}+\sin ^{2} \theta d \phi^{2}\right)
$$

Thus, we have $V_{4}=\frac{1}{2} \pi^{2}$ and $\eta=3$. The corresponding $\tilde{\mu}$ is given by

$$
\frac{1}{\tilde{\mu}}=192 \mu \lambda^{4}
$$

For the case with negative cosmological constant $2 \Lambda=-30 \lambda^{2}$, the internal space is Einstein with $R_{i j}=-6 g_{i j}$, together with the condition (7). If we allow the internal space to be non-compact, such an example can be easily found. We can use the non-compact anti- $\mathbb{C P}^{2}$ metric, obtained by letting $\xi \rightarrow \mathrm{i} \xi$ and $d s^{2} \rightarrow-d s^{2}$ in (12). The resulting action is then given by (9) but with $6 \lambda^{2} \rightarrow-6 \lambda^{2}$. The parameter $\mu$ is still given by (13).

If we insist that internal manifolds must be compact, the negative cosmological constant makes it impossible to find an explicit metric to satisfy (7), since there can be no globally homogenous compact manifold with negative cosmological constant. However, equations of motion deal with only local properties, and a manifold can be locally homogeneous even though it is not globally. An example of this type is the two-dimensional Riemann surfaces with high genuses. There is no reason why a similar phenomenon could not arise in four dimensions.

If the cosmological constant in $D=7$ vanishes, the internal space $d s_{4}^{2}$ is then Ricci-flat. Since there can be no non-trivial Ricci-flat homogeneous space even locally, the lowerdimensional theory cannot inherit any topological term. Thus the cosmological constant appears to be crucial for topologically massive gravity to be embedded consistently in $D=7$. 
In a generic bosonic theory, the coupling constants $\mu, \nu$ in $D=7$ are unrelated; $\mu$ is continuous whilst $\nu$ is quantized by the homotopy condition [8]. Since only the $\mu$ term would yield the topological term in $D=3$, it follows that there is no quantization condition for the mass parameter in $D=3$. However, for theories where $\mu$ and $\nu$ are related, the quantization condition in $D=7$ then provides a quantization condition for $D=3$. In particular, as was pointed out in [8], topological terms in seven dimensions arise naturally in supergravity from the $S^{4}$ reduction of M-theory. In this case $\mu$ and $\nu$ are related to form the anomaly polynomial for M5-brane [9, 10, 11]. The corresponding mass parameter in $D=3$ is then quantized.

To see this in detail, let us write the relevant terms in the $D=11$ supergravity action.

$$
S=\frac{1}{2 \kappa_{11}^{2}} \int R * \mathbb{1}-\frac{1}{2} * F_{(4)} \wedge F_{(4)}-\frac{1}{6} A_{(3)} \wedge\left(F_{(4)} \wedge F_{(4)}+12 \kappa_{11}^{2} T_{3} X_{(8)}\right)+\text { more }
$$

where

$$
X_{(8)}=\frac{1}{192(2 \pi)^{4}}\left(\frac{1}{4} d \Omega_{1}^{(7)}-d \Omega_{2}^{(7)}\right),
$$

and $T_{3}$ is the M2-brane tension, given by

$$
T_{3}=\frac{1}{(2 \pi)^{2} \ell_{p}^{3}}
$$

We have introduced the Planck length $\ell_{p}$, related to $\kappa_{11}$ by $2 \kappa_{11}^{2}=(2 \pi)^{8} \ell_{p}^{9}$.

Eleven-dimensional supergravity admits an $\mathrm{AdS}_{7} \times S^{4}$ vacuum solution, given by

$$
\begin{aligned}
d s_{11}^{2} & =d s_{A d S_{7}}^{2}+Q^{2 / 3} d \Omega_{4}^{2}, \\
F_{(4)} & =3 Q d \Omega_{(4)} .
\end{aligned}
$$

The $\mathrm{AdS}_{7}$ radius is $2 Q^{1 / 3}$. In other words, we have $\lambda=\frac{1}{2} Q^{-1 / 3}$. Here the constant $Q$ is related to the M5-brane charge, which is quantized as $Q=N \pi \ell_{p}^{3}$, where $N$ is the number of M5-branes.

Neglecting the high-order correction terms, the reduction on the $S^{4}$ with fixed breathing mode gives rise to $D=7$ pure gravity with a cosmological constant, namely

$$
S=\frac{1}{2 \kappa_{7}^{2}} \int\left(R+30 \lambda^{2}\right) * \mathbb{1},
$$

where

$$
\kappa_{7}^{2}=\frac{\kappa_{11}^{2}}{Q^{4 / 3} \omega_{4}} .
$$

Here $\omega_{4}=\frac{8}{3} \pi^{2}$ is the volume of a unit $S^{4}$. Note that the theory has a negative cosmological constant. We now consider the reduction including the $X_{(8)}$ as well. The resulting action is 
that of seven-dimensional topological gravity (1) with

$$
\mu=\frac{(2 \pi)^{2} \ell_{p}^{5}}{2^{8}(\pi N)^{1 / 3}}, \quad \nu=-4 \mu .
$$

The homotopy condition associated with the $\nu$ term, discussed in [8], is given by

$$
\frac{64 \pi^{4} \nu}{2 \kappa_{7}^{2}}=\frac{\pi N}{24}=2 \pi n
$$

for integer $n$. Thus we have

$$
N=48 n
$$

Utilizing the previous reduction from $D=7$ to $D=3$, we can now embed $D=3$ topological gravity in M-theory. The parameters in three dimensions can be derived from the M-theory parameters, given by

$$
2 \kappa_{3}^{2}=\frac{6 \pi^{6} \ell_{p}}{(\pi N)^{8 / 3} V_{4}}, \quad \ell=\frac{2}{\sqrt{3}}(\pi N)^{\frac{1}{3}} \ell_{p}, \quad \frac{1}{\tilde{\mu}}=\frac{\pi^{4} \eta \ell_{p}}{2^{5}(\pi N)^{5 / 3} V_{4}} .
$$

The central charges of the boundary conformal field theory were computed in [12, 13], and they can now be expressed in terms of M-theory parameters:

$$
\begin{aligned}
& c_{L}=\frac{12 \pi \ell}{\kappa_{3}^{2}}\left(1-\frac{1}{\tilde{\mu} \ell}\right)=\frac{8 V_{4}}{\sqrt{3} \pi^{2}} N^{3}\left(1-\frac{\pi^{2} \eta}{2^{6} V_{4}} \frac{1}{N^{2}}\right), \\
& c_{R}=\frac{12 \pi \ell}{\kappa_{3}^{2}}\left(1+\frac{1}{\tilde{\mu} \ell}\right)=\frac{8 V_{4}}{\sqrt{3} \pi^{2}} N^{3}\left(1+\frac{\pi^{2} \eta}{2^{6} V_{4}} \frac{1}{N^{2}}\right) .
\end{aligned}
$$

An interesting observation is that the quantity $c_{L}-c_{R}=-\eta N /(4 \sqrt{3})$ depends only on the quantized charge $N$ and the topological charge $\eta$. Therefore, it is invariant under any continuous perturbation, which is consistent with the fact [6] that $c_{L}-c_{R}$ does not change under the holographic RG flow. On the other hand, the quantity $c_{L}+c_{R} \sim N^{3} V_{4}$ suggests that the breathing scalar mode may be turned on by the RG flow.

We now examine the consistency and the validity of our reduction. There are more higher-order correction terms in $D=11$. It is clear that the type of $A \operatorname{Tr}\left(F^{2}\right) \operatorname{Tr}\left(\Theta^{2}\right)$ gives no contribution to the equations of motion with our reduction ansatz. If all the $R^{4}$ terms were known in $D=11$, we would expect that our dimensional reduction procedure would give rise to higher-derivative supergravity in three dimensions. It would be of great interest to investigate whether there exists a limit for which the Lorentz Chern-Simons term dominates. The explicit demonstration can be difficult because not all the $R^{4}$ terms have been known. The fact that TMG can be supersymmetrized in its own right with the minimum supersymmetry suggests that such a truncation to TMG may exist.

To summarize, we have demonstrated that topologically massive gravity in $D=3$ can be obtained from topological gravity in $D=7$ by the consistent Kaluza-Klein reduction on 
an internal Einstein manifold with the Pontryagin four-form satisfying (7). This procedure can be easily generalize to establish a recursive reduction relation. Topological gravities exist in $4 k+3$ dimensions, with $(k+1)$ topological terms. The $k$ terms can be related to those in $4 k-1$ dimensions by $\Omega^{(4 k+3)}=\Omega^{(4 k-1)} \wedge \operatorname{Tr}\left(\Theta^{2}\right)$, and the extra one is given by $d \Omega^{(4 k+3)}=\operatorname{Tr}\left(\Theta^{2(k+1)}\right)$. Thus all but the extra one can be reduced and give rise to all the lower-dimensional topological terms.

A physically more relevant observation is that topologically massive gravity can also be embedded in M-theory, with the mass parameter $\tilde{\mu}$ now determined by the number of M5branes, which has to be large for the truncation approximation to be valid. The condition for chiral topological gravity, namely $\tilde{\mu} \ell=1[3]$, now becomes

$$
\frac{\eta}{V_{4}}=\frac{2^{6} N^{2}}{\sqrt{3} \pi^{2}}
$$

where $\eta$ is the Pontryagin instanton number and $V_{4}$ is the volume of the internal Einstein four-manifold that satisfies $R_{i j}=-6 g_{i j}$ and (7). It is of great interest to investigate whether such a manifold could arise mathematically. The embedding suggests that the dual conformal field theory of three-dimensional topological gravity is the six-dimensional conformal field theory of M5-brane wrapped on the Einstein four-manifold. This proposal is consistent with the leading behavior of the central charges in (24) since the degree of freedom of M5 branes is expected to proportion with $N^{3}$ for large $N$.

\section{Acknowledgement}

We are grateful to Nikolay Bobev, Gary Gibbons, Yi Pang, Chris Pope, and Ergin Sezgin for useful discussions. Z.L.W. is supported in part by grants from the Chinese Academy of Sciences, a grant from 973 Program with grant No: 2007CB815401 and grants from the NSF of China with Grant No: 10588503 and 10535060.

\section{References}

[1] M. Banados, C. Teitelboim and J. Zanelli, "The Black hole in three-dimensional spacetime," Phys. Rev. Lett. 69, 1849 (1992) arXiv:hep-th/9204099.

[2] S. Deser, R. Jackiw and S. Templeton, "Topologically massive gauge theories," Annals Phys. 140, 372 (1982) [Erratum-ibid. 185, 406.1988 APNYA,281,409 (1988 APNYA,281,409-449.2000)]. 
[3] W. Li, W. Song and A. Strominger, "Chiral gravity in three dimensions," JHEP 0804, 082 (2008) [arXiv:0801.4566 [hep-th]].

[4] E.A. Bergshoeff, O. Hohm and P.K. Townsend, "Massive gravity in three dimensions," Phys. Rev. Lett. 102, 201301 (2009) arXiv:0901.1766 [hep-th]]; R. Andringa, E.A. Bergshoeff, M. de Roo, O. Hohm, E. Sezgin and P.K. Townsend, "Massive 3D Supergravity," Class. Quant. Grav. 27, 025010 (2010) arXiv:0907.4658 [hep-th]].

[5] D. Dalmazi, "Unitarity of spin-2 theories with linearized Weyl symmetry in $D=$ $2+$ 1," Phys. Rev. D80 085008 (2009) arXiv:0908.1954[hep-th]]; D. Dalmazi and E.L. Mendonca, "A new spin-2 self-dual model in $D=2+1$," JHEP 0909011 (2009)[arXiv:arXiv:0907.5009 [hep-th]].

[6] K. Hotta, Y. Hyakutake, T. Kubota, T. Nishinaka and H. Tanida, "Left-Right Asymmetric Holographic RG Flow with Gravitational Chern-Simons Term," Phys. Lett. B680, 279(2009). arXiv:0906.1255[hep-th]]

[7] R. Percacci and E. Sezgin, "One Loop Beta Functions in Topologically Massive Gravity," arXiv:1002.2640 [hep-th].

[8] H. Lü and Y. Pang, "Topological Gravity in Seven Dimensions," arXiv:1001.0042 [hep-th]].

[9] M.J. Duff, James T. Liu and R. Minasian,, "Eleven-dimensional origin of string-string duality: A One loop test," Nucl. Phys. B452, 261 (1995) arXiv:hep-th/9506126.

[10] J. A. Dixon, M. J. Duff and E. Sezgin, "The coupling of Yang-Mills to extended objects," Phys. Lett. B279, 265 (1992) arXiv:hep-th/9201019.

[11] L. Alvarez-Gaumé and P. Ginsparg, "The structure of gauge and gravitational anomalies," Ann. Phys. 161, 423 (1985) [Erratum-ibid. 171, 233 (1986)].

[12] J.D. Brown and M. Henneaux, "Central charges in the canonical realization of asymptotic symmetries: an example from three-dimensional gravity," Commun. Math. Phys. 104, 207 (1986).

[13] P. Kraus and F. Larsen, "Holographic gravitational anomalies," JHEP 0601, 022 (2006) arXiv:hep-th/0508218. 